\title{
In-Country Acute Flaccid Paralysis Surveillance Review, Nasarawa State, Nigeria, 2017
}

\author{
Maureen O. Anyanwü ${ }^{* 1}$, Ndubuisi Akpuh ${ }^{2}$ and Adewole Adefisoye ${ }^{3}$ \\ 'Nigeria Field Epidemiology and Laboratory Training Programme/ Oyo State Ministry of Health, Ibadan, Nigeria; ${ }^{2}$ Department of \\ Public Health, Ministry of Health, Rivers State, Portharcourt, Nigeria; ${ }^{3}$ African Field Epidemiology Network, Asokoro, Nigeria
}

\section{Objective}

In August, 2017, we conducted a peer review evaluation of the reported high stool adequacy and Non-polio Acute Flaccid Paralysis (AFP) rates of the World Health Organisation (WHO) verified AFP cases, in order to estimate and establish concordance for both surveillance core indicators in Lafia and Nasarawa Egon LGAs in Nasarawa State.

\section{Introduction}

Nigeria is the only polio endemic country in Africa. Four (4) WPV1 cases were confirmed in 2013 after two years of silence. Nigeria has a strong polio programme characterized by innovative and forward driven strategies, despite several challenges of which surveillance is one of the driving forces. Near perfect surveillance core indicators reported over the past twelve (12) months across certain states and Local Government Areas (LGAs) were issues of concern, given security challenges among others. In August, 2017, we conducted a peer review evaluation of the reported high stool adequacy and Non-polio Acute Flaccid Paralysis (AFP) rates of the World Health Organisation (WHO) verified AFP cases, in order to estimate and establish concordance for both surveillance core indicators in Lafia and Nasarawa Egon LGAs in Nasarawa State.

\section{Methods}

The LGAs to be visited and AFP cases reported within ninety (90) days and verified to be true and adequate prior to peer review were selected. Any person with strong surveillance knowledge and skill, working in Nigeria with the government or partner agencies and involved in surveillance was identified as a peer reviewer, trained and deployed to the LGAs. Reviewers were not deployed to their geo-political zones where they work under routine conditions. Data was collected by visiting the residence of the respective AFP cases and eliciting responses, using a structured interviewer -administered peer review checklist. Data was collated, analysed using Microsoft Excel 2010 and interpreted accordingly. The causes of incoherence were identified and presented to the LGA Disease Surveillance and Notification Officers (DSNOs) and State authority. An improvement plan which would be monitored and evaluated was elaborated. The AFP surveillance data base for discordant AFP cases was updated with the data generated from the peer review.

\section{Results}

Of the nineteen (19) AFP cases reviewed, 63.2\% (12/19) were females. The mean Age of the total AFP case patients was 3 years (SD 3.4). In Lafia LGA, eight (8) AFP cases were verified and all were true AFP cases and adequate. In Nasarawa Egon LGA, eleven (11) cases were verified, $54.5 \%(6 / 11)$ were true AFP cases and 90.9\% (10/11) were found to be adequate. The major causes of the gaps identified include mothers/caregivers dividing collected stool specimen sample to make for two (2) stool samples meant to be collected 24 hours apart for case investigation. This was due to failure on the part of the LGA DSNOs to either inform the mothers/caregivers or underscore the importance of appropriate stool collection. The inability of the surveillance focal officers to adequately identify/differentiate other disease conditions that mimic AFP and persistence of residual paralysis (in Non-polio AFP cases) in $5(45.5 \%)$ cases were also identified in Nasarawa Egon LGA. This was as a result of the lack of referral to the next level for physiotherapy care.

\section{Conclusions}

In Nasarawa Egon LGA, there were discordances in the reported AFP performance core indicators. They include inadequate stool sample, wrong classification of AFP cases and persistence of residual paralysis in Non-polio AFP cases. We therefore, recommend that the WHO State team should re-orient the LGA DSNOs on proper stool specimen collection for case investigation. Also, the LGA DSNOs should sensitize parents/caregivers on appropriate protocol of stool specimen collection and advise them on referral to the next level of care.

\section{Keywords}

AFP; Peer Review; Nasarawa State

\section{*Maureen O. Anyanwu}

E-mail: maureenanyanwu23@yahoo.com 